\title{
Actores y procesos en los convenios urbanísticos para Puerto Norte en Rosario. Notas sobre lo público y lo privado
}

Feld, Natalia

Arquitecta UBA. Magíster en Economía Urbana de la Universidad Torcuato Di Tella. Doctoranda en el Programa de Estudios Urbanos de la Universidad Nacional de General Sarmiento. Pertenencia Institucional: Universidad Nacional de General Sarmiento.

nfeld@campus.ungs.edu.ar

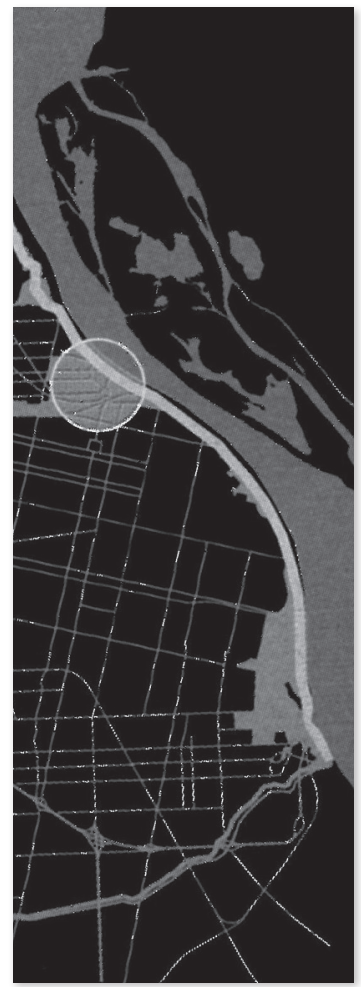

http://dx.doi.org/10.30972/crn.31315777 


\section{Actores y procesos en los convenios urbanísticos para Puerto Norte en Rosario. Notas sobre lo público y lo privado}

\section{Resumen}

Puerto Norte puede analizarse como un Gran Proyecto Urbano y como una de las intervenciones de política urbana más relevante en la ciudad de Rosario. Parte de la bibliografía que estudió estos proyectos encontró en los instrumentos y en las posibilidades de captación de plusvalía urbana las alternativas para contrarrestar posibles procesos regresivos producto de su implementación. Sobre la base de esos resultados, este texto se propone revisitar los instrumentos para, a través del análisis de su proceso de formulación e implementación, examinar las modalidades según las cuales el entramado de actores opera desde sus recursos, estrategias y capacidades diferenciales, en los ámbitos de concertación generados. La posibilidad de centrar la mirada en los actores y procesos permite iluminar nuevos matices sobre cómo son las fronteras porosas que se dirimen entre la esfera pública y la privada, que además se modifican a lo largo del tiempo.

\section{Palabras clave}

Grandes proyectos urbanos; instrumentos; Puerto Norte; acción pública; actores sociales.

\section{Actors and processes in the urban agreements for Puerto Norte in Rosario. Notes on the public and the private}

\section{Abstract}

Puerto Norte may be analysed as a large-scale urban project and as one of most relevant urban policy interventions in Rosario City. Part of the bibliography that studied these projects, found in the instruments, and in the possibilities of capturing urban surplus value, the alternatives to counter possible regressive processes, product of its implementation. Upon these results, this paper endeavours to revisit instruments, through the analysis of their formulation and implementation process, to examine the modalities according to which the actors network operates from its own resources, strategies and differential capabilities, in the areas of agreement generated. The possibility of focusing the scope on actors and processes allows to illuminate new nuances about how the porous borders that are settled between the public and private spheres are, which also changes with time.

\section{Keywords}

Large-scale urban project; instruments; Puerto Norte; public action; social actors.

\section{Atores e processos \\ nos acordos urbanos para \\ Puerto Norte em Rosário. \\ Notas sobre o público e o privado}

\section{Resumo}

Puerto Norte pode ser analisado como um Grande Projeto Urbano e como uma das intervenções de política urbana mais relevantes na cidade de Rosário. Parte da bibliografia que estudou esses projetos encontrou nos instrumentos, e nas possibilidades de captura da mais-valia urbana, as alternativas para neutralizar possíveis processos regressivos decorrentes de sua implantação. Com base nesses resultados, este texto se propõe a revisitar os instrumentos a fim de, por meio da análise de seu processo de formulação e implementação, examinar as modalidades segundo as quais a rede de atores opera a partir de seus recursos, estratégias e capacidades diferenciais, nas áreas de convênio geradas. A possibilidade de focalizar o olhar nos atores e processos, permite iluminar novas nuances sobre como se estabelecem as fronteiras porosas que se estabelecem entre as esferas pública e privada, que também mudam com o tempo.

\section{Palavras-chave}

Grandes projetos urbanos, instrumentos, Puerto Norte, ação pública, atores sociais. 


\section{Introducción ${ }^{1}$}

El 8 de septiembre del año 2005, el Concejo Municipal de la Ciudad de Rosario aprobó por unanimidad la ordenanza correspondiente al "Plan Especial 2. ${ }^{\text {da }}$ Fase del Centro de Renovación Urbana Raúl Scalabrini Ortiz Puerto Norte”, e inauguró un nuevo ciclo en la formulación de políticas públicas urbanas en esa ciudad. El objeto de esta ordenanza era la refuncionalización de cien hectáreas de terrenos ferroportuarios, localizados sobre la costa norte de la ciudad. Por su escala y sus características, la intervención de Puerto Norte habilitada por la ordenanza puede inscribirse en el marco de lo que la bibliografía caracterizó como Grandes Proyectos Urbanos (GPU) y, dentro de estos, los denominados Water Front. En este escenario, lo local y lo global, lo público y lo privado adquirieron nuevas significaciones.

En el debate internacional, parte de la bibliografía analizó a los GPU como la expresión más acabada de los nuevos modos de territorialización del capitalismo globalizado (MouLAERT ET AL., 2005). Desde este enfoque, los proyectos condensan las nuevas lógicas de anclaje territorial y de relaciones con el Estado, producto, a su vez, no solo de los nuevos modos de producción y consumo, sino también del rol que asumen las ciudades en este proceso (Pinson, 2011). Desde esta tradición analítica, el Estado es analizado como conjunto indiferenciado, y las políticas públicas como producto de la sumisión de las agendas urbanas a los requerimientos del capitalismo globalizado (FAinstein, 1991; ORUETA \& FAinsTEIN, 2008). En este contexto, los instrumentos formulados ad hoc fueron entendidos como condición necesaria para garantizar las condiciones de su ejecución (SWYNGEDouw ET AL., 2002; SALeT \& Gualini, 2006).

Esta perspectiva de análisis fue ampliamente utilizada en las investigaciones sobre los GPU en Argentina (Etulain, 2009; Cuenya \& Corral, 2011; Galimberti, 2016). En este contexto, los instrumentos - entendidos como los documentos jurídicos formulados por el Estado para la regulación del desarrollo urbano - fueron visualizados como objeto de estudio privilegiado por sus capacidades para contrarrestar procesos regresivos producto de su implementación. Particularmente en los estudios sobre los GPU y los instrumentos, BEATRIZ CUENYA encuentra en las posibilidades de adaptación de estos últimos los elementos centrales para, por un lado, generar "instancias de negociaciones y acuerdos entre los distintos actores públicos y privados involucrados" (CUENYA, 2012, p. 35), y, por el otro, desde sus capacidades
1. Este texto presenta una primera puesta a punto de materiales, en el contexto de una investigación más amplia que se está llevando adelante en el marco del Programa de estudios Urbanos de la de la Universidad Nacional de General Sarmiento. 
de captar las rentas generadas por la acción de los estados municipales, definir los modos de redistribución del excedente que estos generan, para evitar que sea apropiado por los inversores privados (CUENYA, 2012). En estas capacidades de los instrumentos, junto con la orientación política del gobierno, la autora sitúa la diferenciación en las posibilidades de resolución de las tensiones y conflictos entre intereses, los cuales, a su vez, no son homogéneos y se modifican según el contexto (CUENYA, 2012).

En el caso de Puerto Norte, los instrumentos fueron examinados tanto desde su posibilidad de captación de plusvalía (Cuenya et al., 2004; Cuenya \& Pupareli, 2006; Bragos \& Mazzaro, 2011) como desde su potencialidad de redistribución (Peinado et al. 2018; Peinado, Barenboim, Lagarrigue, \& Nicastro, 2018; Terraza et al., 2015; Galimberti, 2016; Leite et al., 2020; Pontoni \& FERNÁNDEZ, 2015; Rosentein ET AL., 2017). Es de señalar que muchas de las reflexiones y debates que se suscitaron en torno de estos temas a principios de este siglo fueron tributarias de las recomendaciones formuladas por los organismos internacionales de crédito y del Lincoln Institute of Land Policy (Feld, 2015).

Frente a esta línea de investigación, interesa el aporte de Pinson, quien —en continuidad con los trabajos de LE GALES sobre gobernanza urbana- propone nuevos matices que permiten analizar al proyecto urbano como producto de las transformaciones del capitalismo globalizado, pero también como producto de la multiplicidad de intereses públicos y privados que, atravesados por las disputas políticas e ideológicas, estructuran un entramado de actores que los define. En síntesis, como resultado de las transformaciones en las formas de acción pública (Pinson, 2011). Esta noción resulta particularmente pertinente para el abordaje del objetivo propuesto, en tanto permite correr el acento de la esfera institucional, para situar los actores estatales en relación con otros interlocutores (THOENIG, 1997; LAscomunEs \& Le GaLÉs, 2014). Se trata de una noción gestada en Francia en los años 80, al calor de los cuestionamientos sobre el rol del Estado, en un contexto de debates sobre la participación y la descentralización en la formulación de políticas públicas que mostraba nuevas formas de articulación entre lo público y lo privado (Novick, 2009). Esta visión se inscribe, a su vez, en los estudios que colocaron al Estado en primer plano dando cuenta de sus heterogeneidades y coaliciones en el interior de su estructura (SкостоL, 1989; OszLaK \& O’DonnelL, 1995). En última instancia, no se trata de pensar al Estado en términos monolíticos, sino de saber “quiénes son el Estado" (Menazzi \& Jajamovich, 2019, p. 19). 


\section{Actores y procesos en los convenios urbanísticos para \\ Puerto Norte en Rosario. Notas sobre lo público y lo privado}

Sobre la base del resultado de las investigaciones que analizaron esta problemática en Argentina, y a partir de las posibilidades que otorga la noción de acción pública, este texto se propone revisitar los instrumentos poniendo el foco en el proceso de formulación e implementación de los Convenios Urbanísticos ${ }^{2}$ en relación con Puerto Norte. A grandes rasgos se coincide con la bibliografía, en la disparidad de recursos y competencias, producto del nuevo anclaje territorial del capitalismo globalizado. No obstante, la posibilidad de centrar la mirada en los actores ${ }^{3}$ y procesos permite dar cuenta de las fronteras porosas que se dirimen entre la esfera pública y la privada que, por otro lado, se modifican a lo largo del tiempo. Desde esa perspectiva, se trata de examinar las modalidades según las cuales el entramado de actores ${ }^{4}$ opera, al tiempo que estos se construyen como tales, desde sus recursos, estrategias y capacidades diferenciales.

Para posibilitar este abordaje se adoptó una metodología de carácter cualitativo a partir de un estudio de caso y se recurrió al análisis documental centrado en normativas, documentos públicos, transcripciones taquigráficas del Consejo Deliberante de la Ciudad de Rosario, informes financieros de las empresas desarrolladoras, archivos periodísticos y fuentes secundarias. Asimismo, se trabajó con entrevistas en profundidad semiestructuradas a informantes claves ${ }^{5}$. De manera complementaria, el proceso de formulación de los Convenios Urbanísticos ${ }^{6}$ se analizó a partir de la metodología utilizada por Alicia Mateos para la Ordenanza de Urbanización de Rosario. Según afirma, este tipo de análisis

Posibilita el reconocimiento de los actores involucrados, la identificación de sus intereses, la caracterización de sus relaciones (las alianzas y los enfrentamientos), la visualización de las estrategias aplicadas y de los recursos instrumentales que los distintos actores ponen en juego para el logro de sus objetivos (MatEos, 2003, p. 136).
2. Entendido como un instrumento jurídico que permite formalizar instancias de acuerdo para la realización de proyectos específicos entre el Estado Municipaly desarrolladores privados, en el que se establecen las contraprestaciones que debe asumir cada uno (LEVIN, 2012).

3. En este trabajo, la referencia a los actores se realiza a partir de los cargos y los roles que desempeñaron a lo largo del proceso.

4. En el presente trabajo, se analizan los actores que intervinieron desde la esfera pública y la privada. A pesar de que a lo largo del proceso hubo organizaciones sociales que se expresaron sobre de lo construido en Puerto Norte, tales como "Tren para todos" $y$ "Grito de Malvinas", estas no tuvieron intervención directa a lo largo del proceso. Sí se registraron resistencias ante las alternativas para la urbanización de la UG3, donde actualmente se localizan un asentamiento y una empresa recuperada; sin embargo, este caso no es incorporado en el análisis aquí realizado.

5. En función de la limitada extensión del artículo, solo se incluyeron parte de los materiales de trabajo considerados indispensables para respaldar el argumento. Hasta el momento se realizaron doce entrevistas. Parte de ellas a funcionarios y exfuncionarios públicos que permitieron dar cuenta de las miradas, las estrategias y los conflictos que se encontraron por detrás del proceso. Por otra parte, se entrevistó a docentes de la UNR para dar cuenta del rol que ocuparon los técnicos en la construcción de la nueva mirada sobre la arquitectura y la ciudad. Teniendo en cuenta que la investigación está en curso, aún resta la realización de nuevas entrevistas.

6. El análisis se realiza a partir de la restitución del proceso por el que atravesaron la Ordenanza Básica de Puerto Norte, las Ordenanzas Complementarias correspondientes a los Convenios Urbanísticos allí previstos y aquellas que posteriormente modificaron las condiciones iniciales de realización. 
Esto es posible en tanto los instrumentos se constituyen en "objetos en torno a los cuales las tensiones aludidas se manifiestan con particular virulencia, constituyendo, por otra parte, las instancias materiales en las que estas tensiones asumen una forma concreta y a partir de las cuales es posible analizarlas” (Mateos, 2003, p. 136).

Este texto se organiza en tres apartados, según una periodización cronológica-problemática. El primer momento, que corresponde al período 1991-2003, permitirá retomar los antecedentes del proyecto para inscribir el proceso de aprendizaje en el que se construyeron los actores y se desplegaron las estrategias orientadas a garantizar las condiciones de posibilidad. El segundo, entre los años 2003-2008, permitirá dar cuenta del ciclo de disidencias y negociaciones en relación con el proceso de construcción de las capacidades diferenciales de actuación. Por último, el tercer momento, que inicia en el año 2008, permitirá dar cuenta de los factores que incidieron en el cambio de rumbo por el que atravesó el proceso.

\section{Las condiciones de posibilidad: aprendizaje y construcción de actores}

Figura 1. Localización de Puerto Norte en la ciudad de Rosario Fuente: Plan Urbano Rosario 20072017, Municipalidad de Rosario

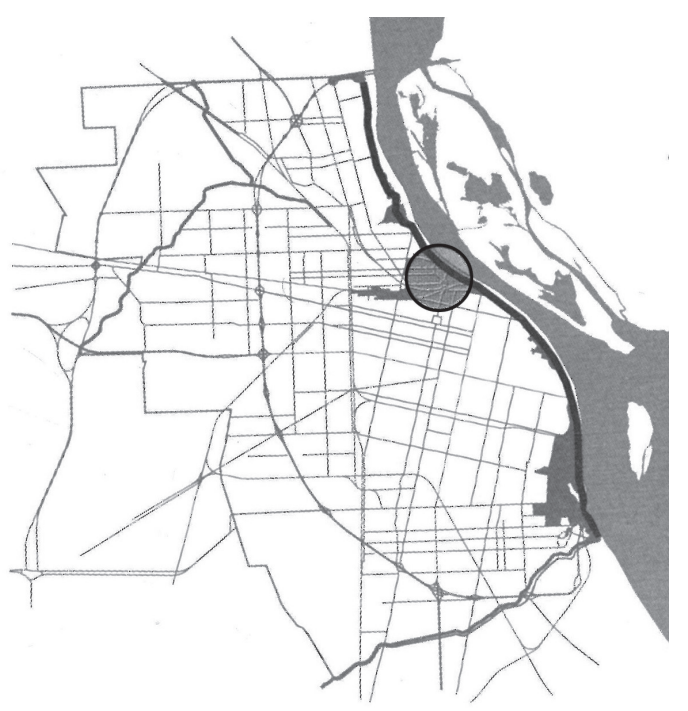

La posibilidad de iluminar algunos de los antecedentes sobre Puerto Norte permite examinar el origen de las lógicas técnicas, políticas y territoriales que lo determinaron. El sector donde actualmente se desarrolla Puerto Norte, históricamente destinado a las actividades portuarias, se encuentra localizado sobre la costa del río Paraná, al norte de la ciudad. Las primeras iniciativas para trasladar el antiguo puerto de Rosario hacia la zona sur de la ciudad se remontan al primer Plan Regulador de Rosario publicado en 1935 (Rigotri, 2011), el Plan Regulador de 1952, posteriormente el del 1967/8 y el Código Urbano de 1968. Sin embargo, a partir del regreso de la democracia en 
el año 1983, las alternativas de refuncionalización de la costa en general y de Puerto Norte en particular asumieron nuevas significaciones, producto en parte de los debates internacionales sobre el proyecto urbano y las posibilidades multiplicadoras que suponía su implementación.

En este contexto comenzaron a configurarse las especificidades de la planificación urbana en Rosario. En primer lugar, la continuidad partidaria del socialismo en la administración municipal entre los años 1989 y 2019. En segundo lugar, la consolidación de los equipos técnicos de la Secretaría de Planeamiento, a partir de una particular articulación entre ámbitos académicos y de gestión (Mateos, 2003). Y, por último, el modo en que los debates epistemológicos sobre la planificación estratégica y el proyecto urbano fueron incorporados en la gestión (JajAmovich, 2009; GaLIMBERTI, 2015).

De esta manera, se comenzaron a desarrollar los primeros debates morfológicos sobre las alternativas de refuncionalización de los terrenos ferroportuarios. Particularmente, en el marco de la formulación del Plan Director publicado en el año 1991, se realizó el "Estudio Particularizado del Área de Puerto Norte", publicado en el año 1987, y el "Seminario Internacional de Proyectos Urbanos. Área de Puerto Norte”, realizado en el año 1991. Este último se constituyó en un significativo parteaguas de estas transiciones, pues allí se reunieron las primeras reflexiones proyectuales para el sector, en clave Proyecto Urbano. En la participación de los equipos locales es posible comenzar a restituir las trayectorias de actores que posteriormente serán claves en el devenir del proceso. Interesa particularmente dar cuenta del trabajo coordinado por Isabel Martínez de San Vicente, quien, junto a Fernández de Luco $^{7}$, había realizado sus estudios de posgrado en Italia ${ }^{8}$. Este equipo de trabajo se encontró conformado por Mirta Levín, María Eugenia Bielsa, Ricardo Kingland y Pablo Ábalos, entre otros. La restitución de sus trayectorias individuales y políticas permite explicar el rol que asumieron en el devenir del proceso.

Esos debates morfológicos sobre las alternativas de refuncionalización en Puerto Norte tuvieron su correlato en la promulgación de una serie de instrumentos normativos orientados a iniciar el proceso de desafectación de las actividades portuarias, acordar las condiciones de realización con las entidades que tenían dominio sobre estos terrenos y, por último, definir la zonificación como área de reserva. En este contexto, en el año 1996, se aprobó la "Primera Fase del Centro de Renovación Urbana Scalabrini Ortiz". Posteriormente, en el
7. Fernández de Luco se desempeñó primero como director de la Oficina del Plan Director y posteriormente como secretario de Planificación del Municipio de Rosario.

\section{Su participación en} el CURDIUR y la UNR contribuyó al traslado de las alternativas planteadas por "la arquitectura de la ciudad" a los debates locales. 


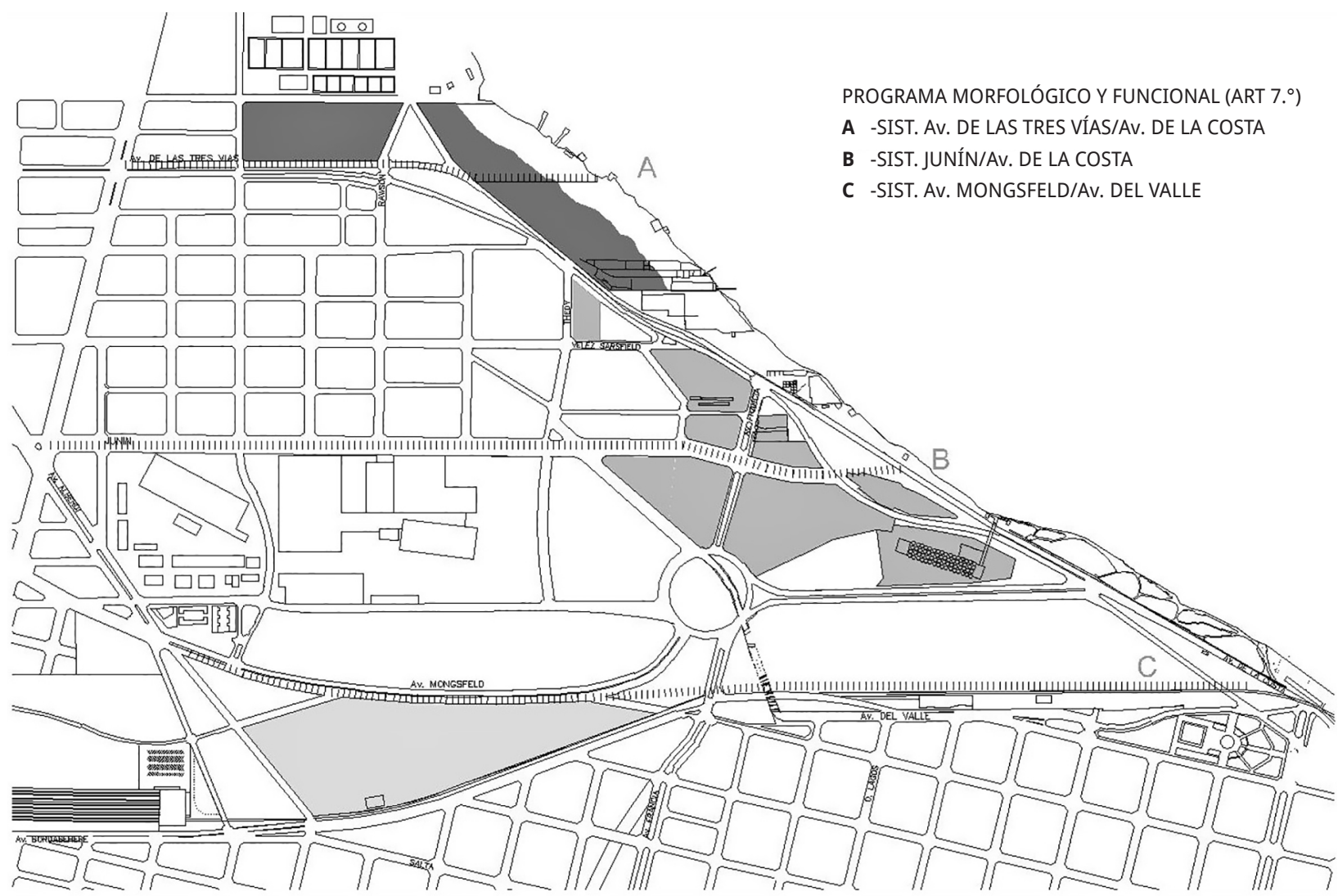

Figura 2. Programa Morfológico Funcional Proyecto, según los tres ejes o sistemas urbanos. Fuente: Proyecto de Ordenanza retirado, Bases del Concurso nacional de Anteproyectos Ideas para la Elaboración del Plan Particularizado para la Segunda Fase del Centro de renovación Urbana Scalabrini Ortiz

año 2001, se presentaría el primer anteproyecto de ordenanza correspondiente a la segunda fase de Puerto Norte, que no se aprobó por no haber obtenido los consensos necesarios.

Tal como es posible mostrar en el plano (figura 2), el proyecto de ordenanza retirado se encontraba estructurado a partir de, por un lado, los "sistemas urbanos" que establecía el programa morfológico y los usos generales admitidos; por el otro, los "distritos” que 
definían las condiciones de subdivisión y los indicadores edilicios. La definición previa de las condiciones de realización condicionaba la gestión ante la heterogeneidad de intereses privados que se encontraban operando en Puerto Norte. Mientras un sector comenzaba a intervenir en relación con las potenciales rentas inmobiliarias del proyecto, el otro comenzaba a oponer resistencias judiciales al traslado de las actividades portuarias.

Dentro del primer grupo de empresas se encontraba Inversiones y Mandatos, que en el año 2000 había adquirido la Unidad de Gestión (UG) N. ${ }^{\circ} 5$ y acordado los indicadores de un ambicioso desarrollo inmobiliario (CuENYA ET AL., 2012). A partir del cambio de gestión en el año 2003, el presidente del directorio, Aldo Lattuca, comenzó a exigir la sanción de la Ordenanza Básica de Puerto Norte, a la que había quedado supeditada la habilitación final de su emprendimiento. La segunda empresa fue IRSA, que en el año 2003 había comenzado las obras del shopping en el terreno perteneciente a la "Primera Fase del Centro de Renovación Urbana Scalabrini Ortiz”. Este desarrollo fue llevado adelante con la participación de un ex secretario de Planeamiento, quien, desde sus capacidades adquiridas en la gestión municipal, participaba del equipo de profesionales encargado del proyecto. Finalmente, su posibilidad de realización permitió no solo vislumbrar las primeras transformaciones materiales en el paisaje de Puerto Norte (BARENBoim, 2016), sino que, además, reforzó ante el resto de las empresas la potencialidad de las rentas futuras.

En contraposición al interés sobre las rentas inmobiliarias, dentro del segundo grupo que continuaba desarrollando actividades portuarias se encontraba Servicios Portuarios, que había comenzado a operar en Puerto Norte a principio de los años 90 con terminales de embarque en la denominada Unidad Portuaria III (UG N. ${ }^{\circ}$ 6). Esta empresa se resistió al traslado de sus actividades portuarias a través de litigios judiciales.

Con el objetivo de redefinir las condiciones preestablecidas en el proyecto de ordenanza del año 2001 y acordar las condiciones de realización con cada desarrollador de manera independiente, la Secretaría de Planeamiento lo retiró de su tratamiento en el Concejo Deliberante y promovió la realización del "Concurso Nacional de Anteproyectos e Ideas para la elaboración del Plan Particularizado para la $2^{\text {da }}$ Fase del Centro de Renovación Urbana Scalabrini Ortiz” en el año 2004. De esta manera, era posible otorgar legitimidad al nuevo esquema de gestión, a las decisiones morfológicas, y de manera simultánea, iniciar el proceso de valorización del suelo. 
El primer premio del concurso (figura 3) fue otorgado al arquitecto rosarino Juan Munuce, quien, desde su amplia experiencia, proponía una secuencia de edificios articulados por espacios públicos y privados orientados a promover la integración con la ciudad existente. Una vez realizado el concurso, la estrategia para llevar adelante el nuevo proyecto de ordenanza fue, por una parte, contratar a Juan Munuce para traducir las premisas proyectuales a la letra del nuevo proyecto de ordenanza. Por otra parte, contratar a Jorge Doncel, quien, desde su amplia trayectoria en desarrollos inmobiliarios en la ciudad (BAREnBoim, 2013), brindaba asesoramiento sobre las condiciones de rentabilidad de la propuesta.

Figura 3. Primer Premio Concurso Nacional de Anteproyectos e Ideas. Autor: Arq. Juan Munuce. Fuente: archivo arquitecto Juan Munuce

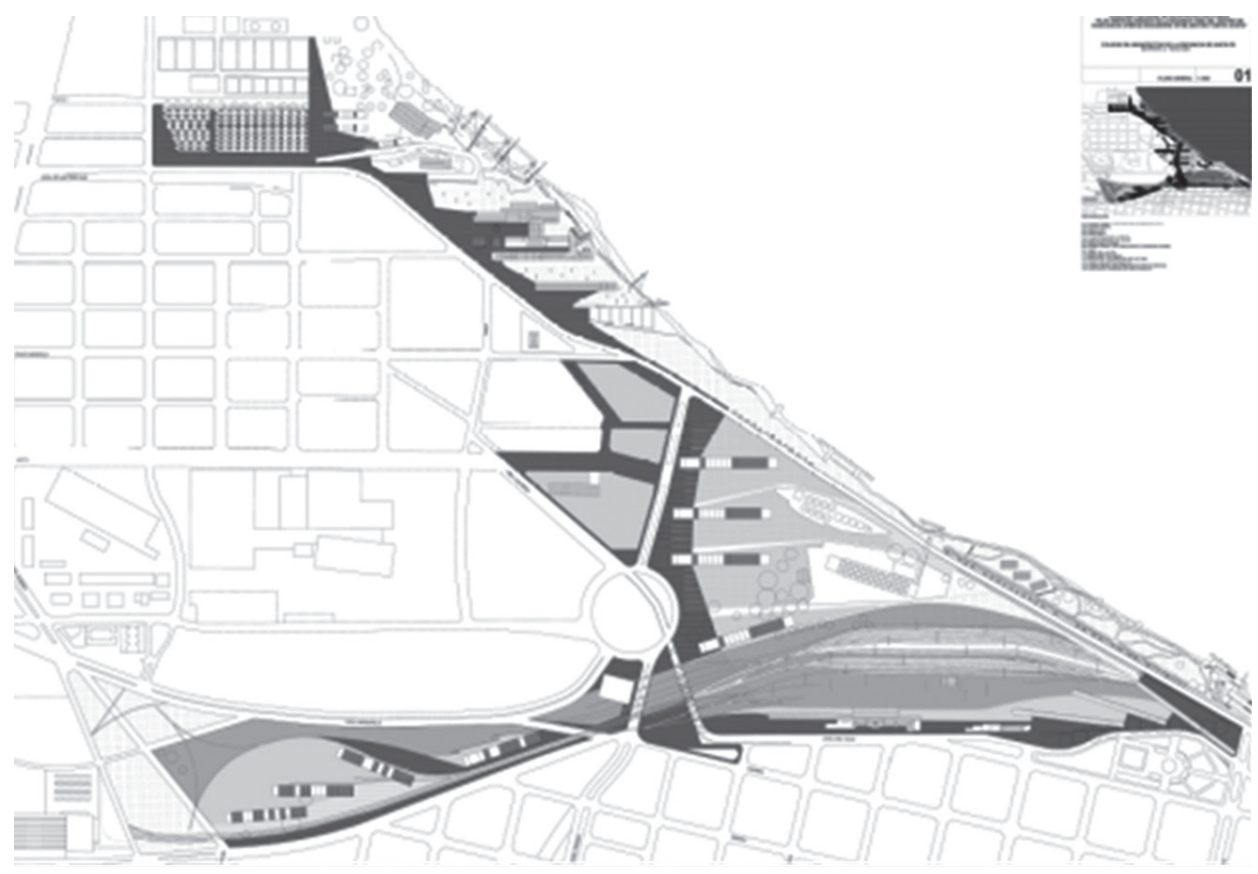


De esta manera, la Secretaría logró adquirir capacidades proyectuales e inmobiliarias para construir los consensos requeridos y logró la aprobación de la Ordenanza N. ${ }^{\circ} 7892 / 2005$, correspondiente al "Plan Especial $2^{\text {da }}$ Fase del Centro de Renovación Urbana Raúl Scalabrini Ortiz. Puerto Norte”. Esta sectorizó el área en siete polígonos denominados Unidades de Gestión (UG), ahora sí "en función de la propiedad de la tierra o de las diferentes modalidades de organización entre propietarios" (MCR, 2005). Simultáneamente, en el marco técnico administrativo, según las normas urbanísticas particulares, Puerto Norte se inscribió como Plan Especial (Ordenanza Básica) e incorporó la división en Planes de Detalle (Ordenanzas Complementarias).

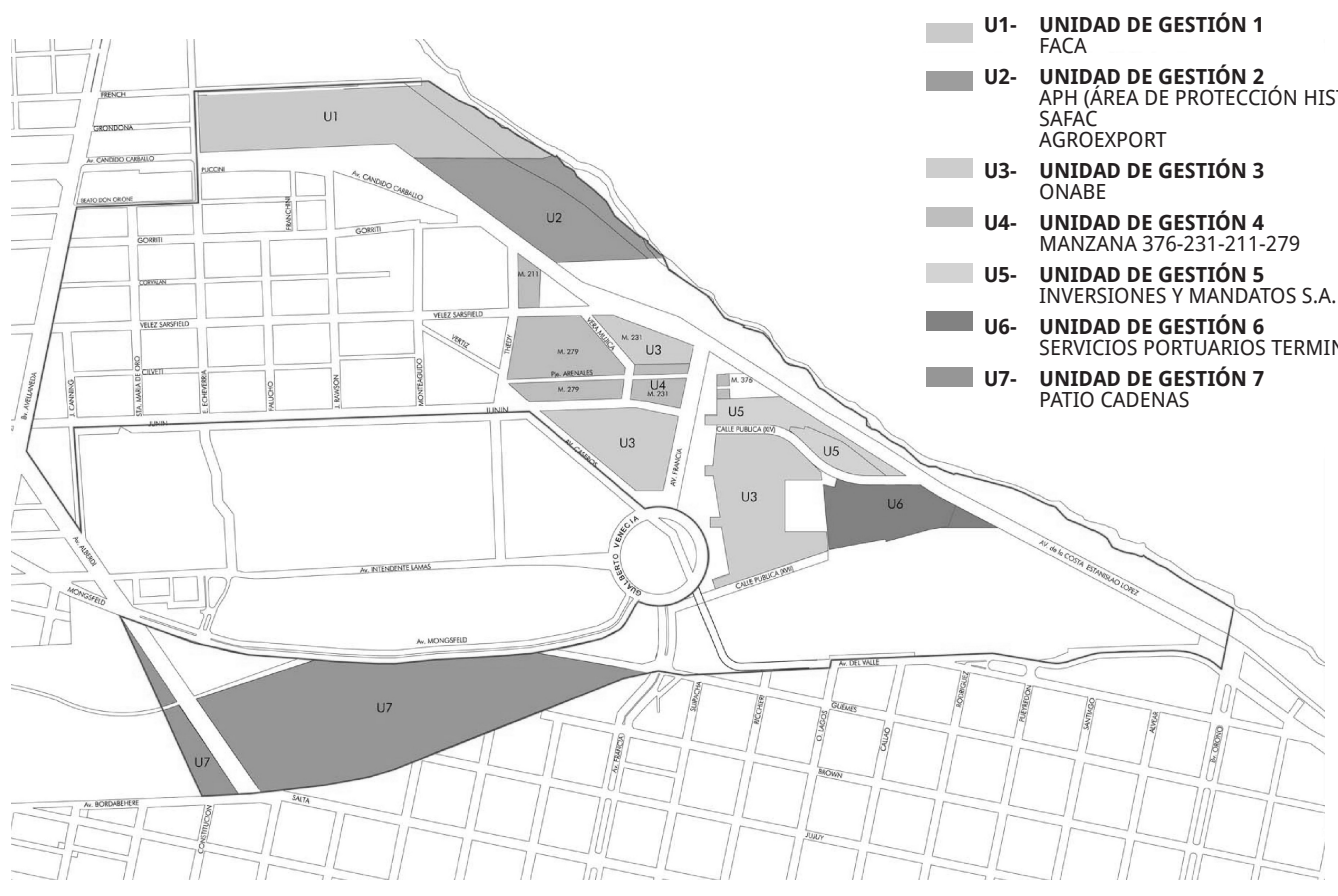

Figura 4.

Estructuración a partir de Unidades de Gestión. Fuente: $M C R$, Ordenanza7892/05 
9. La Ordenanza de Urbanización $N .^{\circ}$ 6492/97 posibilitó la implementación de dos herramientas indispensables para Puerto Norte: el "Programa de Urbanización Integral", que establece las condiciones para la habilitación de tierra vacante con destino urbano, que regula la donación de espacios verdes y la provisión de servicios de infraestructura, y el "Convenio Urbanístico en suelo Urbanizable”, que posibilita la generación de instancias de acuerdo con los desarrolladores para establecer el modo y el plazo en que estas condiciones serán ejecutadas.
Es decir que el Municipio adoptó el siguiente esquema, según la Ordenanza de Urbanización ${ }^{9}$, para la gestión de Puerto Norte: la Ordenanza Básica establecía los lineamientos generales del Plan Especial; las Ordenanzas Complementarias, las condiciones de urbanización referidas a la morfología y los indicadores edilicios de cada UG. Estos quedaban formalizados en un Plan de Detalle a través de un Acta Acuerdo. Ambos constituían el Convenio Urbanístico.

Esta diferenciación en sectores utilizando los principios del Convenio Urbanístico, indicados en el plano (figura 4), además de posibilitar la rearticulación de los procesos público-privados de toma de decisiones, le permitió a la Secretaría de Planeamiento incorporar nuevas obligaciones por fuera de la Ordenanza de Urbanización, a través de la figura de "servidumbre administrativa de uso público". Esta permitía incorporar nuevos espacios de acceso público en terrenos de dominio privado. La coexistencia de ambos le otorgó a la Secretaría de Planeamiento nuevas posibilidades de negociación y la suficiente flexibilidad para acordar las condiciones de urbanización de manera particular con cada desarrollador (KoZAK \& FELD, 2018).

En síntesis, la Ordenanza Básica de Puerto Norte puede ser comprendida como corolario de la extensa tradición de técnicos y políticos en la recuperación del frente costero según los alcances del proyecto urbano. En ese contexto, y ante la creciente disponibilidad de capitales orientados a la inversión inmobiliaria, intereses públicos y privados comenzaron a alinearse bajo las premisas del proyecto. De esta manera se inicia un período de aprendizaje, mediante el cual tanto las empresas privadas como los agentes municipales, en su proceso de construcción, comenzaron a adquirir nuevas capacidades de operación.

Desde la esfera de lo público, la estrategia fue adquirir competencias en relación con las condiciones de rentabilidad que manejaba el sector privado. Para ello, las posibilidades de concertación implícitas en la figura del Convenio Urbanístico posibilitaron garantizar las condiciones proyectuales del concurso y las de rentabilidad de los desarrollos. Este esquema a su vez permitió otorgarle celeridad al proceso y traccionar los intereses de aquellas empresas que continuaban oponiendo resistencias. De manera recíproca, las empresas desarrolladoras requerían recursos para acceder a los contactos y a la información necesaria para operar en los ámbitos de negociación que la misma ordenanza establecía. Estas "puertas giratorias" entre la esfera pública y la privada comenzaron a signar el devenir del proceso. 


\section{La puesta en marcha: disidencias y negociaciones}

Hasta aquí fue posible iluminar algunos de los factores que permitieron la puesta en marcha de Puerto Norte. En el presente apartado, se aborda el momento en el cual se desarrollaron de las negociaciones de los Convenios Urbanísticos, a los fines de dar cuenta de la relevancia del esquema de gestión en la generación de los ámbitos de negociación y de las estrategias asumidas por los actores para adquirir capacidades diferenciales de operación.

En un primer momento, situado entre los años 2005 y 2006, el proceso no encontró mayores resistencias y, en función de los consensos ya construidos, se aprobaron los Convenios Urbanísticos de la UG N. ${ }^{\circ} 5$ (2005) y los correspondientes a la UG N. ${ }^{\circ} 2$ Sector 1 y a la Manzana 407 de la UG N. ${ }^{\circ} 4$ (2006). En un segundo momento, ubicado durante el transcurso del año 2008, se aprobaron la UG N. ${ }^{\circ} 2$ - Sector 2, las UG N. ${ }^{\circ} 1$ y UG N. ${ }^{\circ} 6$ y las manzanas nomencladas como 279 y 376 de la UG N. ${ }^{\circ} 4$. Este se encontró atravesado por una nueva tensión producto de las elecciones celebradas en año 2007 y la nueva composición de la oposición en el Concejo Deliberante, que comenzó a desplegar acciones en bloque oponiendo resistencias al proceso. A partir del análisis de los debates parlamentarios, es posible restituir algunas de las tensiones y negociaciones que se encontraron por detrás de la suscripción de los Convenios Urbanísticos. En este contexto, la voz opositora de la concejala María Eugenia Bielsa ${ }^{10}$ permite dar cuenta del modo en que los debates académicos fueron recuperados e instalados en la esfera legislativa.

La argumentación "científica", que promovía la implementación de políticas redistributivas, a partir de las posibilidades de reglamentación de la figura del Convenio Urbanístico, se transformó en uno de los ejes discursivos de la oposición. En ese escenario la tensión que enfrentó la Secretaría de Planeamiento fue doble. Por un lado, negociar con celeridad las condiciones de realización de cada Convenio Urbanístico, en consonancia con los tiempos cortos exigidos por de los desarrolladores. Por otro lado, construir los acuerdos en el Concejo Deliberante para aprobar de las Ordenanzas Complementarias y dar comienzo a las obras. En este contexto, la figura del Convenio Urbanístico posibilitó consolidar los ámbitos de negociación para el procesamiento de las condiciones de ejecución de las Unidades de Gestión y la construcción de los consensos para su realización.
10. Su trayectoria como arquitecta urbanista, al igual que Levin, Kingsland y Ábalos, se inició en el área de investigación en la Universidad Nacional de Rosario. Se trata de figuras que venían participando en los debates proyectuales sobre Puerto Norte desde el Seminario Internacional de Proyectos del año 1991. 
No obstante, el desarrollo de estos ámbitos adquirió distintos grados de complejidad, en función de las exigencias de cada desarrollador. Las estrategias desplegadas por aquellas empresas que no pertenecían al ámbito local tuvieron un denominador común: la posibilidad de adquirir capacidades diferenciales de actuación no solo sobre las particularidades del mercado inmobiliario en la ciudad de Rosario, sino también sobre los canales de acceso a los contactos y a la información para operar en dichos ámbitos de negociación.

La primera estrategia fue replicar aquella asumida por IRSA para el desarrollo del shopping y contratar a profesionales con capacidades adquiridas en la administración municipal, para la ejecución de sus proyectos. Por un lado, Inversiones y Mandatos (UG N. ${ }^{\circ}$ 5) contrató a quien se había desempeñado primero como subsecretario y luego como secretario de Planeamiento en el año 1999. Por otro lado, Ingenconser desarrolladora de Ciudad Ribera (UG N. ${ }^{\circ}$ 2- Sector 2) contrató al arquitecto que, luego de dirigir la Secretaría de Planeamiento entre los años 1995 y 1999, asumió distintos cargos de gestión. Es decir que las capacidades adquiridas desde la gestión municipal eran requeridas por el sector privado para operar en los ámbitos de negociación. En este punto, interesa retomar los postulados de Menazzi y JajAmovich que, a partir de las relaciones entre saberes y prácticas, encuentran en las trayectorias de los actores la clave para relativizar las relaciones binarias entre lo público y lo privado (Menazzi \& JАјАмоvich, 2019, p. 20). La segunda estrategia fue asociarse con empresas menor escala, pero con trayectoria en el ámbito inmobiliario local. En el caso TGLT, desarrolladora de Forum en la UG N. ${ }^{\circ} 2$ Sector 1, se asoció con las empresas Edgardo Berman y Lamelas. En cuanto a IRSA, para el desarrollo de Condominios del Alto en la manzana 407, se asoció con las empresas Fundar y Rosental. Ambas estrategias permitieron la adquisición de capacidades diferenciales para operar en los ámbitos de negociación construidos alrededor de la figura del Convenio Urbanístico.

En síntesis, estos “ámbitos de negociación” se constituyeron en la arena de combate donde se dirimieron las coaliciones entre los intereses de las empresas que requerían determinados indicadores urbanísticos para sus desarrollos inmobiliarios y los de la Secretaría de Planeamiento, que imponía condiciones y compensaciones para aprobarlos. Desde la esfera municipal, la estrategia se basó en consolidar las condiciones de liderazgo ya construidas durante el momento de la gestación y, de esta manera, constituirse 
como único interlocutor frente a los actores inmobiliarios. Aquí el esquema de gestión adoptado fue fundamental para otorgarle celeridad al proceso, construir los consensos en el marco de los debates parlamentarios y acordar las condiciones de realización con los desarrolladores inmobiliarios. Desde la esfera privada, las estrategias adoptadas posibilitaron que las empresas, en su proceso de construcción, pudieran adquirir capacidades diferenciales de operación en aquellos ámbitos de negociación que se estructuraron por detrás de las alternativas del Convenio Urbanístico. Estas capacidades otorgaron acceso tanto al conocimiento sobre las lógicas de funcionamiento del gobierno y del mercado inmobiliario local como a la red de contacto e información requerida para operar en dichos ámbitos.

\section{Un cambio de rumbo: nuevos actores y reglas de juego}

En los apartados anteriores fue posible, a partir de la restitución del proceso de formulación y puesta en marcha de los Convenios Urbanísticos, dar cuenta del modo en que, en su proceso de construcción, los actores fueron adquiriendo capacidades diferenciales para operar en los ámbitos de negociación. En el presente apartado se propone dar cuenta de los factores que incidieron en el nuevo rumbo que adquirió el proceso, producto de, por un lado, el cambio de gobierno en el año 2011 y la consecuente pérdida de protagonismo de la Secretaría de Planeamiento y, por el otro, el cambio en la política económica nacional implementada a partir del año 2016, que modificó la ecuación económica de los desarrollos inmobiliarios en relación con rentabilidades y facilidades que comenzó a otorgar el mercado financiero.

El cambio de rumbo en la gestión del proyecto permitió el despliegue de nuevas estrategias por parte de los actores privados que ya se habían construido y consolidado alrededor de las alternativas del proyecto. En esta orientación resulta paradigmático el proceso por el que atravesó el Convenio Urbanístico correspondiente a las UG N. ${ }^{\circ} 1$ y UG N. ${ }^{\circ} 6$, suscripto con Servicios Portuarios. En el primer apartado, fue posible dar cuenta de su estrategia inicial para resistir al traslado de las actividades portuarias a partir de demandas judiciales. Cuando estas se resolvieron a favor del Estado y debió dejar de funcionar, diversificó sus actividades de negocios y se incorporó en el tablero de la competencia inmobiliaria. Con este objetivo, siendo propietaria de la UG N. ${ }^{\circ}$ 6, adquirió en el año 2005 los terrenos correspondientes a la UG N. ${ }^{\circ} 1$ (CUENYA, 2012). Estos factores hicieron de la 
11. Mirta Levin, comunicación personal, 7 de noviembre de 2019. Entrevista realizada por Natalia Feld.

\section{Fernández Prieto había} formado parte de las empresas desarrolladoras que realizaron las Torres Yacht y de Zencity en Puerto Madero.

13. Al no verse afectados los indicadores edilicios establecidos en el Convenio Urbanístico del año 2008, no fue necesaria la suscripción de un nuevo Convenio Urbanístico.

14. Inicialmente se podía subdividir en cinco áreas de intervención constituyendo cada una de ellas una unidad de ejecución de proyecto. $L a$ modificación del año 2013 subdividió a la Unidad de Gestión en seis unidades de ejecución de proyecto. negociación con Servicios Portuarios la más compleja de resolver. Al respecto, la entonces secretaria de Planeamiento afirmó:

Para establecer un equilibrio entre los distintos propietarios de los predios de Puerto Norte se fija como marco de negociación un FOT entre 2 y 2,5. La diferencia entre estos valores (que fueron establecidos con ese margen de flexibilidad para permitir un manejo más adecuado de las morfologías) se equilibra con las exigencias de obras. La Unidad 1 y 6, al pertenecer a una misma firma, fueron tratadas en forma conjunta de modo tal que los indicadores urbanísticos definidos se repartieran en las dos unidades de ejecución sin que la firma propietaria del predio se vea más favorecida que otros actores de Puerto Norte. En este caso la negociación fue más difícil que con el resto, dado que existían reclamos judiciales previos que debían contemplarse a los efectos de que la firma del Convenio Urbanístico sirviera, también, para dar por finalizados esos reclamos judiciales ante el municipio. ${ }^{11}$

Una vez resuelta la complejidad de la negociación, en el mes de noviembre del año 2008, el Concejo Deliberante aprobó la Ordenanza Complementaria para la urbanización de ambas UG. Las estrategias desplegadas por Servicios Portuarios para consolidarse en el mercado inmobiliario se centraron en la construcción de sociedades con empresas que tuvieran expertise en este tipo de desarrollos.

En este contexto, la primera estrategia asumida fue asociarse con el estudio de Fernández Prieto, con vasta experiencia en desarrollos en Puerto Madero ${ }^{12}$, para la construcción de las Torres Maui en un sector de la UG N. ${ }^{\circ}$ 6. La segunda fue asociarse con TGLT, para el proyecto Metra en UG N. ${ }^{\circ} 1$. Esta empresa, al igual que la anterior, contaba con una amplia trayectoria en desarrollos inmobiliarios en ciudades como Buenos Aires y Montevideo, pero, además, tenía antecedentes en Puerto Norte con la realización de Forum. A su vez, la estrategia de TGLT fue impulsar el desarrollo con un nuevo proyecto a cargo de un famoso estudio británico, para lo cual requirió la modificación de la propuesta morfológica y de la subdivisión del suelo que estableció el Convenio Urbanístico inicial. ${ }^{13}$ Para ello, en el año 2013, se aprobó una segunda ordenanza con las modificaciones que el nuevo proyecto requería. Tal como se desprende de la comparación gráfica de ambas (figuras 5 y 6), la segunda otorgaba mayores posibilidades de subdivisión para su posterior comercialización. ${ }^{14}$ 


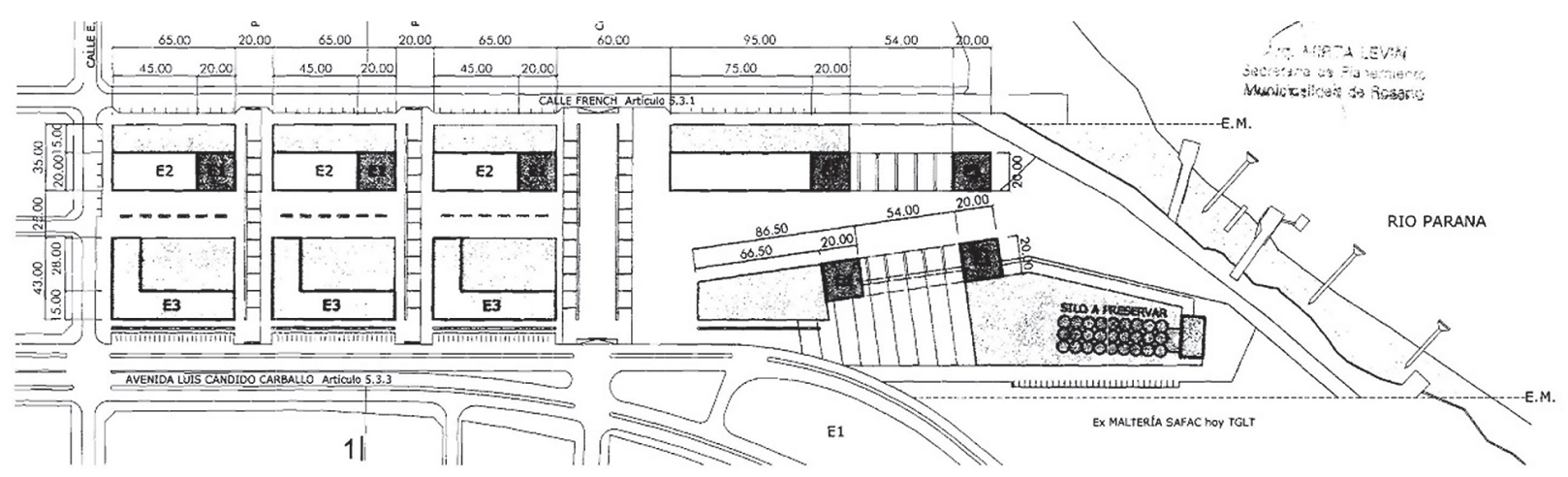

Figura 5. Plan de Detalle Unidad de Gestión 1. Fuente: MCR, Ordenanza 8320/2008

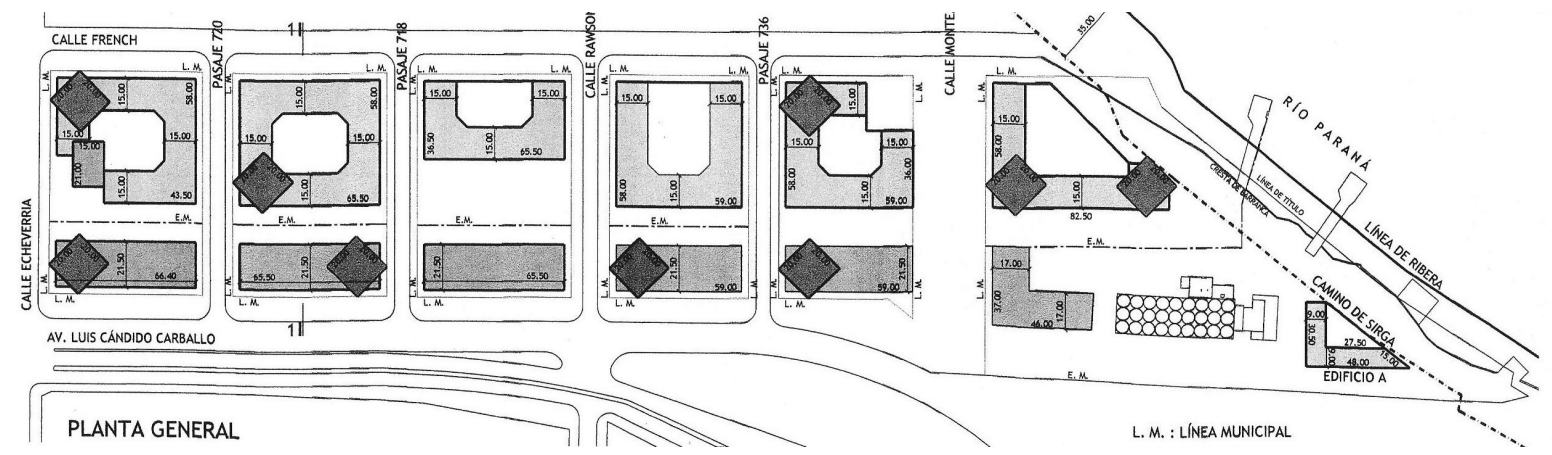

Figura 6. Modificación de Plan de Detalle Unidad de Gestión 1. Fuente: MCR, Ordenanza 9047/13

Ahora bien, en el año 2018, cuando todavía no había empezado la construcción de la primera torre, TGLT compró la empresa Caputo y redefinió su intervención en Puerto Norte. En una primera instancia, redujo a la mitad el proyecto original. Esto se debió, según un comunicado de la empresa, "a la nueva coyuntura económica, que permitió la aparición de nuevas formas de financiamiento más atractivas” ${ }^{15}$. Sin embargo, a finales del año 2018 decidió retirarse definitivamente de Puerto Norte, ante lo cual Servicios Portuarios puso en
15. Cita extraída del artículo "TGLT asunción que hará dos edificios en Puerto Norte" (18 de junio de 2006). La Capital. https://www.lacapital.com.ar/ la-ciudad/tglt-anuncio-queno-hara-dos-edificios-puertonorte-n1574036.html 
marcha la tercera estrategia: subdividir la operación en unidades más pequeñas y, a partir de la figura de fideicomiso, incorporar inversores locales de menor escala. Se trata de las empresas BLD, Sancor Seguros y un grupo de inversores privados.

La cuarta estrategia desplegada por Servicios Portuarios fue vender, en el año 2017, el segundo sector de la UG N. 6 a la empresa Turicentro Viajes de Carlos Gianni, para el desarrollo de un hotel de una importante cadena internacional. Las condiciones de rentabilidad que imponía el nuevo proyecto implicaban un aumento significativo de la superficie máxima edificable (de $21.000 \mathrm{~m}^{2}$ a $41.500 \mathrm{~m}^{2}$ ), por lo tanto, al modificarse los indicadores, se requería la suscripción de un nuevo Convenio Urbanístico. Sin embargo, a diferencia del procedimiento utilizado anteriormente, las cargas de urbanización serían acordadas una vez aprobados los nuevos indicadores, por lo que quedaba restringida la capacidad de negociación por parte de la Secretaría. En la comparación gráfica entre la vista del proyecto original y la de la modificación solicitada (figuras 7 y 8), puede observarse la incidencia del edificio destinado al hotel (E3).

Figura 7. Vista

Sur. Fuente: $M C R$,
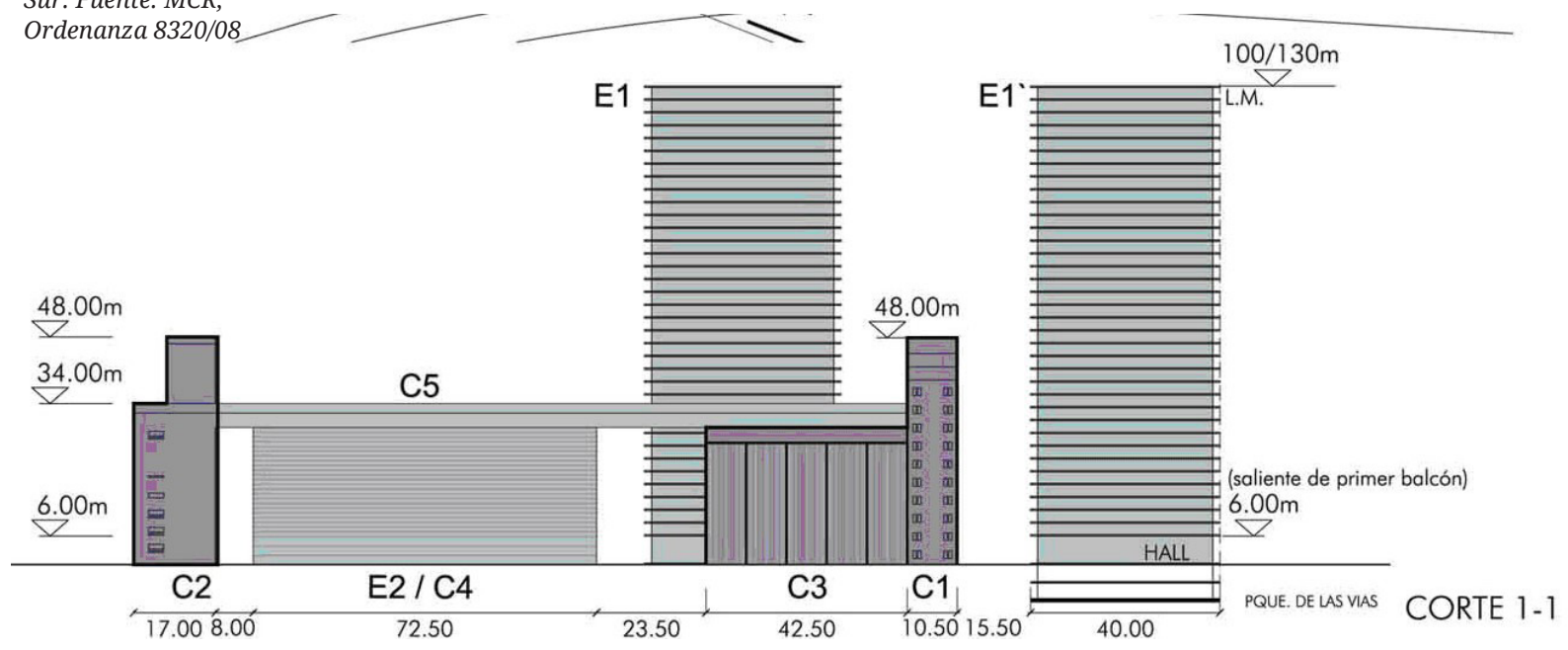


\section{Actores y procesos en los convenios urbanísticos para \\ Puerto Norte en Rosario. Notas sobre lo público y lo privado}

La posibilidad de volver sobre los planteos de la oposición en los debates del Concejo Deliberante nuevamente permite restituir algunas de las tensiones y conflictos que se encontraron por detrás del proceso de negociación. Interesa particularmente dar cuenta de lo expresado por el concejal Monteverde de Ciudad Futura ${ }^{16}$, quien, en continuidad con el papel que había desempeñado María Eugenia Bielsa, ponía en evidencia las controversias del proceso. Al respecto afirmaba:

La empresa Servicios Portuarios hace un convenio con la Municipalidad para desarrollar la Unidad de Gestión 6, la Municipalidad y el Concejo le dan determinados indicadores, Servicios Portuarios, dueño de la tierra, no solamente no cumple con las compensaciones, todavía no construyó ni medio departamento, sino que en el medio vende un pedazo de esos indicadores a este emprendedor que aparece ahora, es decir, sin hacer absolutamente nada, sin poner un peso de inversión vende los indicadores que este Cuerpo le entregó a Servicios Portuarios para hacer un proyecto determinado, ahora dice: “No, ese pedazo no lo voy a hacer” y se lo vendo a este emprendedor" y este emprendedor además tiene la caradurez de decir: "Quiero más metros cuadrados” o sea hoy estamos inaugurando la categoría de "indicador futuro” (CMCR, 29 de junio de 2017).

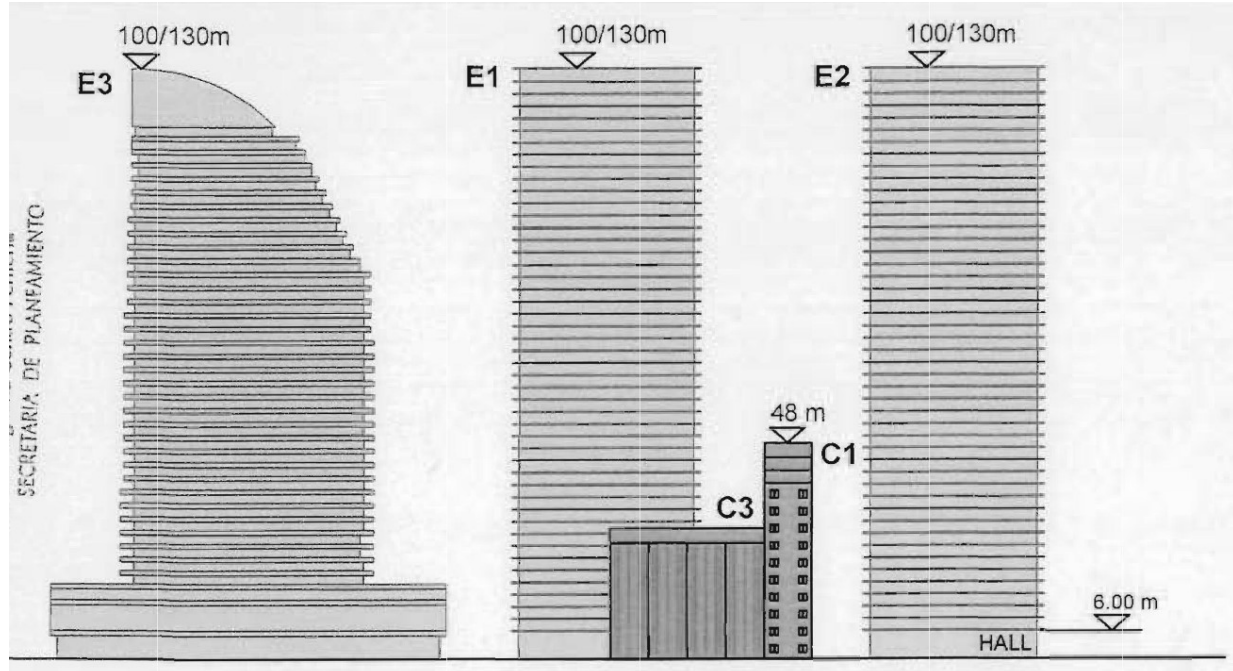

16. Se trata de un partido político que surgió de la Organización Social Giros. En el año 2010, a través de la intervención de María Eugenia Bielsa en el Concejo Deliberante, la organización había logrado la aprobación de la Ordenanza del "ya basta”, que prohibió la figura de los barrios cerrados en la ciudad de Rosario. 
No obstante, el oficialismo, que continuaba teniendo la mayoría reglamentaria, logró la aprobación de la ordenanza mediante la cual se modificó la morfología general, se autorizó la construcción del hotel, se incrementó en casi un 100 \% la superficie máxima edificable y se redefinieron los elementos de protección histórica directa. Finalmente, este desarrollo quedó suspendido producto de procesos judiciales que enfrentaba Carlos Gianni, por lo que el inicio de la obra quedó supeditada su resolución de estos.

En síntesis, este tercer momento mostró un punto de inflexión respecto de los dos anteriores. Por una parte, el cambio de gestión en la Secretaría de Planeamiento debilitó el liderazgo que había sido construido alrededor de los ámbitos de negociación. Estos habían permitido tensionar el intercambio para encauzar los acuerdos que otorgaban cierto grado de cohesión al proceso. Por la otra, y como contracara de la misma moneda, Servicios Portuarios, que había comenzado a construirse en la década del 90 como empresa cerealera, se terminó de consolidar como el actor privado de mayor protagonismo en el tablero inmobiliario de Puerto Norte.

Ahora bien, resulta interesante interrogarse, a más de diez años de aprobación este Convenio Urbanístico con Servicios Portuarios, y considerando el escaso estado de avance de las obras, cuál ha sido el resultado de la nueva correlación de fuerzas en la transformación del territorio.

\section{Notas de cierre}

En la ciudad de Rosario la intervención de técnicos, políticos y académicos en los debates sobre la planificación urbana se encontró signada por una serie de especificidades producto de la continuidad de gobiernos socialistas, la articulación histórica entre saberes técnicos y políticos y el modo en que tanto la planificación estratégica como el proyecto urbano fueron reinterpretados en la realidad local. Estos factores fueron centrales en el proceso mediante el cual la costa se consolidó como territorio proyectual y, por lo tanto, otorgaron las condiciones de posibilidad para que, frente al proceso de reactivación del mercado inmobiliario iniciado en el año 2003, Puerto Norte pudiera pasar de los debates a las transformaciones territoriales. En este contexto, los funcionarios técnicos requerían formular una estrategia orientada a que las "cosas sucedan" y, desde esa perspectiva, los lineamientos morfológicos iniciales podrían ser sostenidos en la medida en que estuvieran garantizadas las condiciones de rentabilidad que lo hicieran factible. 
En ese momento inicial de aprendizaje, en que se estaban construyendo los actores y las competencias para llevar adelante Puerto Norte, los límites entre lo público y lo privado comenzaron a desdibujarse frente a las estrategias de los actores que, en su proceso de construcción alrededor de las múltiples alternativas de negociación intrínsecas en la figura del convenio urbanístico, construyeron las capacidades diferenciales para operar. En un segundo momento de negociación, la clave se situó en el esquema de gestión adoptado por una Secretaría de Planeamiento que, consolidada en su liderazgo, obtuvo la capacidad de construir ámbitos de concertación con cada propietario de manera independiente. A su vez, algunos de ellos encontraron en la experiencia de lo público la posibilidad de adquirir nuevas capacidades para operar en dichos ámbitos. En el tercer momento de inflexión, al modificarse el entramado de actores y lo que estaba en juego, aquellas bases que habían posibilitado la cohesión del proceso se encontraron desarticuladas frente a la pérdida de protagonismo de la Secretaría en sus posibilidades de intervención. Como contracara de la misma moneda, empresas que se habían construido alrededor de las alternativas de Puerto Norte desde sus inicios se terminaron de consolidar a partir de las capacidades diferenciales para operar en el mercado inmobiliario y generar nuevos procesos de valorización progresiva del suelo.

Conceptualmente el abordaje desde la acción pública, recuperado en este texto desde la posibilidad de matizar la existencia de la esfera pública y privada, permitió restituir el entramado de actores, las estrategias y las capacidades diferenciales de operación construidas alrededor los ámbitos de negociación producto de los Convenios Urbanísticos. La mirada procesual permitió mostrar que los actores se construyen como tales a partir de la adquisición de nuevas capacidades diferenciales para operar en dichos ámbitos. Las historias mínimas posibilitaron iluminar las condiciones individuales de la multiplicidad de actores que, en distintos niveles, intervienen en la construcción del territorio.

Finalmente, a partir del abordaje propuesto fue posible, desde lo empírico, sumar miradas a la bibliografía sobre Puerto Norte para, a partir de la posibilidad de superar las visiones polares entre lo público y privado, comenzar a matizar esa distinción y mostrar que la propia noción de entramado de actores se pone en jaque cuando se acerca la lupa y se observa que aquellas fronteras que parecían compactas comienzan a mostrar distintos grados de porosidad. Asimismo, desde esa perspectiva, fue posible analizar el GPU como pieza de un engranaje más amplio, producto del entramado social y político que los 
determinó. En este escenario, el proyecto deja de ser la imagen de las páginas satinadas en las revistas especializadas y se convierte en un objeto más difuso de gestiones y de negociaciones.

Tal vez Puerto Norte -en tanto proyecto urbano que permitió formular algunas hipótesis de trabajo acerca de los procesos y del entramado de actores, para ser probadas en otros casospueda considerarse como un prisma para comprender quiénes y cómo construyen la ciudad.

\section{Referencias bibliográficas}

Barenboim, C. (2013). El mercado de suelo y su ordenamiento en la periferia de las ciudades: El caso de Rosario, Argentina. Teseo \& U. A. Interamericana, Eds.

Barenboim, C. (2016). Cambios socioespaciales en los barrios aledaños a Puerto Norte, Rosario. Cuestión Urbana, 1(1).

Bragos, O., \& Mazzaro, P. (2011). Desarrollo urbano, equidad territorial e instrumentos de recuperación de plusvalías. Proyectos y realizaciones. Primer Congreso Latinoamericano de Estudios Urbanos. Universidad Nacional de General Sarmiento, (2010), 1-20.

CMCR, Concejo Municipal de la Ciudad de Rosario (2017). Versión Taquigráfica 29 de junio de 2017. Modificación ordenanza 8320, Plan Especial 2a ${ }^{\text {a }}$. Fase Centro de Renovación Urbana Scalabrini Ortiz Puerto Norte.

CNAeI-Bases (2004). Colegio de Arquitectos Distrito 2. Bases del Concurso Nacional de Anteproyectos e Ideas para la elaboración del Plan Particularizado para la 2 da Fase del Centro de Renovación Urbana Scalabrini Ortiz. Rosario, 3 de agosto de 2004.

Cuenya, B. (2012). Grandes Proyectos Urbanos, cambios en la centralidad urbana y conflictos de intereses. Notas sobre la experiencia Argentina. En B. Cuenya; P. Novais \& C. Vainer (Eds.), Grandes Proyectos Urbanos. Miradas críticas sobre la experiencia argentina y brasileña. Editorial Café de las Ciudades, Colección Planeamiento.

Cuenya, B. \& Corral, M. M. (2011). Empresarialismo, economía del suelo y grandes proyectos urbanos: el modelo de Puerto Madero en Buenos Aires. EURE (Santiago), 37(1083), 25-45. http://www.scielo.cl/scielo.php?script=sci_arttext\&pi$\mathrm{d}=\mathrm{S} 0250-71612011000200002$ 
Cuenya, B. \& Pupareli, S. (2006). Grandes proyectos como herramientas de creación y captación de plusvalías urbanas. Proyecto Puerto Norte Rosario, Argentina. Medio Ambiente y Urbanización. No. 65, IIED-AL, Buenos Aires.

Cuenya, B. \& Corral, M. M. (2011). Empresarialismo, economía del suelo y grandes proyectos urbanos: el modelo de Puerto Madero en Buenos Aires. EURE (Santiago), 37(1083), 25-45. http://www.scielo.cl/scielo.php?script=sci_arttext\&pi$\mathrm{d}=$ S0250-71612011000200002

Cuenya, B.; González, E., Mosto, G. \& Pupareli, S. (2012). Movilización de plusvalías en un gran proyecto urbano. La experiencia de Puerto Norte, en Rosario. En B. Cuenya, P. Novais \& C. Vainer (Eds.), Grandes Proyectos Urbanos. Miradas críticas sobre la experiencia argentina y brasileña. Café de las Ciudades.

Cuenya, B.; Pupareli, S.; Brunstein, F.; Cascella, H.; Mosto, G.; Di Loreto, M. \& Smolka, M. (2004). Análisis y proposición de métodos de recuperación de plusvalías urbanas generadas por la acción pública municipal. Informe final de Consultoría. Anexo. Municipalidad de Rosario. Servicio Público de la Vivienda. Programa Rosario Hábitat y Centro de Estudios Urbanos y Regionales.

Etulain, J. C. (2009). Gestión urbanística y proyecto urbano. Modelos y estrategias de intervención. Nobuko.

Fainstein, S. S. (1991). Promoting economic development: Urban planning in the united states and great britain. Journal of the American Planning Association, 57(1), 22-33. https://doi.org/10.1080/01944369108975469

Feld, N. (2015). Puerto Norte en Rosario. Actores, instituciones e instrumentos en los Grandes Proyectos Urbanos. (Maestría en Economía Urbana). Universidad Torcuato Di Tella. https://doi.org/10.17227/ted.num19-1049

Galimberti, C. I. (2015). La reinversión del río. Procesos de transformación en la ribera de la Región Metropolitana de Rosario, Argentina. (Tesis doctoral). Universidad Nacional de Rosario, Facultad de Arquitectura, Planeamiento y Diseño.

Galimberti, C. I. (2016). Políticas públicas en el desarrollo de grandes proyectos de reconversión urbana. Caso Puerto Norte en Rosario, Argentina. Cadernos Metrópole, 18(36), 559-582. https://doi.org/10.1590/2236-9996.2016-3612

Jajamovich, G. (2009). La circulación internacional de ideas y estrategias urbanísticas y sus aplicaciones transculturadas: Buenos Aires y Rosario (1976-1993). Universidad Torcuato Di Tella. 
Kozak, D. \& Feld, N. (2018). Grandes proyectos urbanos y su relación con la ciudad: el caso de Puerto Norte (Rosario, Argentina). EURE, 44(133), 187-210. https://doi. org/10.4067/s0250-71612018000300187

Lascomunes, P. \& Le Galés, P. (2014). Sociología de la acción pública. El Colegio de México, Centro de Estudios Demográficos Urbanos y Ambientales.

Leite, C.; Acosta, C.; Militelli, F.; Jajamovich, G.; Wilderom, M.; Bonduki, N. ... Herling, T. (2020). Buenos Aires and Rosario: Large-Scale Urban Projects and the Just City. In Social Urbanism in Latin America (pp. 137-147). https://doi. org/10.1007/978-3-030-16012-8_6

Levin, M. (2012). Los Grandes Proyectos Urbanos. La experiencia de la ciudad de Rosario, Argentina. Revista Digital Café de Las Ciudades, Número 119. Retrieved from http://www.cafedelasciudades.com.ar/planes_119.htm

Mateos, A. (2003). La conciliación de intereses en el proceso de elaboración de los instrumentos urbanísticos. El campo de disputa en torno a la Ordenanza de Urbanizaciones de Rosario. En L. de Queiroz Riveiro \& O. Bragos (Eds.), Territorios en transición. Políticas públicas y transformaciones metropolitanas. (p. 174). Universidad Nacional de Rosario.

MCR (1997). Municipalidad de la Ciudad de Rosario. Ordenanza N. ${ }^{\circ}$ 6492/97.

MCR (2004). Proyecto de Ordenanza retirado, Bases del Concurso Nacional de Anteproyectos e Ideas para la Elaboración del Plan Particularizado para la Segunda Fase del Centro de Renovación Urbana Scalabrini Ortiz.

MCR (2005). Municipalidad de la Ciudad de Rosario. Ordenanza Básica N. ${ }^{\circ}$ 7892/05.

MCR (2005). Municipalidad de la Ciudad de Rosario. Ordenanza Complementaria N. ${ }^{0} 7893 / 05$.

MCR (2006). Municipalidad de la Ciudad de Rosario. Ordenanza Complementaria N. ${ }^{\circ} 8065 / 06$.

MCR (2006). Municipalidad de la Ciudad de Rosario. Ordenanza Complementaria N. ${ }^{\circ} 8080 / 06$.

MCR (2008). Municipalidad de la Ciudad de Rosario. Ordenanza Complementaria N. ${ }^{\circ} 8237 / 08$.

MCR (2008). Municipalidad de la Ciudad de Rosario. Ordenanza Complementaria N. ${ }^{\circ} 8320 / 08$.

MCR (2008). Municipalidad de la Ciudad de Rosario. Ordenanza Complementaria N. ${ }^{\circ} 8297 / 08$. 
MCR (2008). Municipalidad de la Ciudad de Rosario. Ordenanza Complementaria N. ${ }^{\circ} 8359 / 08$.

MCR (2012). Municipalidad de la Ciudad de Rosario. Ordenanza N. ${ }^{\circ} 8973 / 12$.

MCR (2017). Municipalidad de la Ciudad de Rosario. Ordenanza N. ${ }^{\circ}$ 9756/17

Menazzi, L. \& Jajamovich, G. (Eds.). (2019). Saberes Urbanos. Profesionales, técnicos, funcionarios y agencias estatales en la producción de ciudad. Teseo Press.

Moulaert, F.; Rodriguez, A. \& Swyngedouw, E. (2005). Introduction. In F. Moulaert, A. Rodriguez, \& E. Swyngedouw (Eds.). The Globalized City: Economic Restructing and Social Polarization in European Cities. Oxford Geographical and Environmental Studies Series.

Novick, A. (2009). Territorialidad y acción pública en Buenos Aires en la década de 1920. In A. Catenazzi, A. Quintar, M. C. Cravino, N. Da Representacao, \& A. Novick (Eds.), El retorno de lo político a la cuestión urbana (pp. 135-159). Universidad Nacional de General Sarmiento y Prometeo Libros.

Orueta, F. D. \& Fainstein, S. S. (2008). The new mega-projects: Genesis and impacts. International Journal of Urban and Regional Research, 32(4), 759-767. https:// doi.org/10.1111/j.1468-2427.2008.00829.x

Oszlak, O. \& O’ Donnell, G. (1995). Estado y políticas estatales en América Latina: hacia una estrategia de investigación. Redes, 2(4), 99-128.

Peinado, G.; Barenboim, C.; Lagarrigue, P. \& Nicastro, M. (2018). Los convenios urbanísticos en la ciudad de Rosario, Argentina, como instrumentos de gestión del suelo. Análisis de sus impactos en términos de equidad. https://www. lincolninst.edu/pt-br/publications/working-papers/los-convenios-urbanisticos-en-la-ciudad-rosario-argentina-como

Peinado, G.; Barenboim, C.; Nicastro, M. \& Lagarrigue, P. A. (2018). Recuperación de plusvalías urbanas y sus impactos distributivos. Las compensaciones por mayor aprovechamiento urbanístico a raíz de convenios urbanísticos en la ciudad de Rosario, Argentina. Sociedad y Economía, 35, 50-77. https:// doi.org/https://www.doi.org/10.25100/sye.v0i34.6476

Pinson, G. (2011). Urbanismo y gobernanza de las ciudades europeas. Gobernar la ciudad por proyecto. Universitat de València.

Pontoni, S. A. \& Fernández, M. L. (2015). Avances y dificultades en el reconocimiento de los impactos de grandes intervenciones urbanas en Rosario. En XXXIV Encuentro Arquisur. XIX Congreso Ciudades Vulnerables. 
Proyecto o incertidumbre, Facultad de Arquitectura y Urbanismo, Universidad Nacional de La Plata Eje: Investigación (Vol. 162, pp. 1-13).

Rigotti, A. M. (2011). El río como argumento. En Ciudad de Rosario (p. 179). Museo de la Ciudad- Editorial Municipal de Rosario. http://rephip.unr.edu.ar/handle/2133/2646

Rosentein, C.; Chajchir, E.; Molteni, G.; Formica, D.; Ruani, J. \& Ruani, S. (2017). Los grandes proyectos urbanos: modelos de gestión y construcción de lo "público”. http://rephip.unr.edu.ar/bitstream/handle/2133/6856/Rosenstein. pdf?sequence $=3$

Salet, W. \& Gualini, E. (2006). Framing strategic urban projects: Learning from current experiences in European urban regions. In Framing Strategic Urban Projects: Learning from Current Experiences in European Urban Regions. https://doi.org/10.4324/9780203966129

Skocpol, T. (1989). El Estado Regresa al Primer Plano: Estrategias de Análisis en la Investigación Actual. Zona Abierta, 50, 71-122. http://polired.upm.es/index.php/ ciur/article/viewFile/921/936

Swyngedouw, E.; Moulaert, F. \& Arantxa, R. (2002). Neoliberal Urbanization in Europe: Large-Scale Urban Development Projects and the New Urban Policy. Antipode, 34, 542-577.

Terraza, H.; Pons Ginés, B.; Soulier, M. \& Juan, A. (2015). El caso de la recuperación del frente costero del río Paraná en la Ciudad de Rosario, Argentina. Banco Interamericano de Desarrollo. https://www.rosario.gob.ar/ArchivosWeb/ bid.pdf

Thoenig, J. (1997). Política pública y acción pública. Gestión y Política Pública, VI(1), 19-37. 